\title{
Energy efficient resource management in mobile grid
}

\author{
Chunlin $\mathrm{Li}^{*}$ and Layuan $\mathrm{Li}$ \\ Department of Computer Science, Wuhan University of Technology, Wuhan, P.R. China
}

\begin{abstract}
Energy efficient computing has recently become hot research area. Many works have been carried out on conserving energy, but considering energy efficiency in grid computing is few. This paper proposes energy efficient resource management in mobile grid. The objective of energy efficient resource management in mobile grid is to maximize the utility of the mobile grid which is denoted as the sum of grid application utility. The utility function models benefits of application and system. By using nonlinear optimization theory, energy efficient resource management in mobile grid can be formulated as multi objective optimization problem. In order to derive a distributed algorithm to solve global optimization problem in mobile grid, we decompose the problem into the sub problems. The proposed energy efficient resource management algorithm decomposes the optimization problem via iterative method. To test the performance of the proposed algorithm, the simulations are conducted to compare proposed energy efficient resource management algorithm with other energy aware scheduling algorithm.
\end{abstract}

Keywords: Mobile grid, resource management, energy efficient

\section{Introduction}

Mobile grid means that movable wireless devices are integrated into traditional wired grid through wireless channel to share grid resources (CPU power, storage capacity, instrument, devices, data, software, etc.), meanwhile, mobile devices can provide service or resource to grid users, such as PDAs, cellular phones, handsets or wearable computers, laptops with GPS service, mobile service, etc. [1]. Mobile grid can provide end users with grid services such as music, medical, accounting, message alert service, function estimation, data mining, assurance, and so on. Mobile grid may be constructed on current network infrastructure, integrate continually developing wireless network technologies, enrich network contents and software platform function. Mobile grid includes various kinds of mobile devices, and then leads to the grid system more complicated than wired grid system due to the mobile grid node dynamically behavior in the grid system. Mobile grid requires dynamic management of distributed resources, and such management needs to meet application quality requirements and prolong application lifetimes. Mobile grid application' lifetime is determined by available energy on the mobile devices. Mobile devices are battery-driven, and hence operate on an extremely frugal energy budget. Considering energy efficiency in mobile grid is an important challenge.

This paper proposes energy efficient resource management in mobile grid. The objective of energy efficient resource management in mobile grid is to maximize the utility of the mobile grid which is denoted as the sum of grid application utility. The utility function models benefits of application and

\footnotetext{
${ }^{*}$ Corresponding author. E-mail: chunlin74@whut.edu.cn; chunlin74@yahoo.com.cn.
} 
system. By using nonlinear optimization theory, energy efficient resource management in mobile grid can be formulated as multi objective optimization problem. In order to derive a distributed algorithm to solve global optimization problem in mobile grid, we decompose the problem into the sub problems. The proposed energy efficient resource management algorithm decomposes the optimization problem via iterative method. To test the performance of the proposed algorithm, the simulations are conducted to compare proposed energy efficient resource management algorithm with other energy aware algorithm.

The rest of the paper is structured as followings. Section 2 discusses the related works. Section 3 presents energy efficient resource management in mobile grid. Section 4 presents energy efficient resource management algorithm in mobile grid. In Section 5 the simulations are conducted. Section 6 gives the conclusions to the paper.

\section{Related work}

Energy efficient computing has recently become hot research area. Many works have been carried out on conserving energy, but considering energy in grid computing is few. Tao Xie et al. [2] address the issue of allocating tasks of parallel applications in heterogeneous embedded systems with an objective of energy-saving and latency-reducing. They proposed BEATA (Balanced Energy-Aware Task Allocation), a task allocation scheme considering both energy consumption and schedule length, is developed to solve the energy-latency dilemma. Y. Huang et al. [3] present techniques for exploiting intermittently available resources in grid infrastructures to support QoS-based multimedia applications on mobile devices. They integrate power aware admission control, grid resource discovery, dynamic load-balancing and energy adaptation techniques to enable power deficient devices to run distributed multimedia applications. Ziliang Zong et al. [4] design energy efficient scheduling algorithms for parallel applications running on clusters, they propose a scheduling strategy called energy efficient task duplication schedule, which can significantly conserve power by judiciously shrinking communication energy cost when allocating parallel tasks to heterogeneous computing nodes. Tarek A. AlEnawy et al. [5] propose to minimize the number of dynamic failures while remaining within the energy budget. They propose techniques to statically compute the speed of the CPU in order to meet the $(\mathrm{m}, \mathrm{k})$-firm deadline constraints. Kyong Hoon Kim et al. [6] provide power-aware scheduling algorithms for bag of tasks applications with deadline constraints on DVS enabled cluster systems in order to minimize power consumption as well as to meet the deadlines specified by application users. Eunjeong Park et al. [8] designed an entire process of multimedia service composition for mobile computing. Their approach adapts the composition graph and the use of service routing for the context of mobile devices with the support of monitoring components. The works [9-13] mainly deal with resource allocation, QoS optimization in the computational grid and don't consider energy consumption for mobile grid. Narottam Chand et al. [17] propose a utility based cache replacement policy, least utility value (LUV), to improve the data availability and reduce the local cache miss ratio. Markus Aleksy et al. [18] present a generic architecture, which can be used for the development of context-sensitive mobile applications. Vincent Reinhard et al. [19] propose a mechanism for the introduction of application data in the network with respect to CARRIOCAS architecture. P. Hellinckx et al. [18] introduced a new multipurpose Lightweight Grid (LWG) system under the acronym Computational Basic Reprogrammable Adaptive (CoBRA) grid. The methods and contributions of this paper are different from above works. This paper is targeted to manage energy consumption without compromising system's performance in mobile grid. 


\section{Energy efficient resource management in mobile grid}

\subsection{Energy efficient resource management mathematic model}

In mobile grid, energy resources distribution and computation workloads are not balanced within mobile devices. It is important for the mobile grid system to design energy efficient resource management. Firstly, let consider the notations used in the following sections. $e_{i}^{l}$ is the energy obtained by grid application $i$ from energy resource $l . C e_{l}$ is the capacity of energy resource $l$. $C n_{k}$ is the capacity of network resource $k . C c_{j}$ is the capacity of computing power $j . t_{i}^{n}$ is the time taken by the grid application $i$ to complete $n$th job. $P c_{i}^{j}$ is the payments of the grid application $i$ to the computing power j. $P n_{i}^{k}$ is the payments of the grid application $i$ to the network resource $k . P e_{i}^{l}$ is the payments of the grid application $i$ to energy resource $l . B_{i}$ is the expense budget of grid application $i$. $e r_{l}$ is the energy consumption rate of energy resource $l$. $e_{i}^{n}$ is energy dissipation caused by grid application $i$, $\mathrm{s} n$th job. $c q_{i}^{n}$ is the computation task of $i$ th grid application' s $n$th job. $b q_{i}^{n}$ is the transmission task of $i$ th grid application's $n$th job. $e q_{i}^{n}$ is the energy storage task of $i$ th grid application's $n$th job. $n p_{k}$ the price of network resource $k . c p_{j}$ is the price of computing resource $j . e p_{l}$ is the price of energy resource $l$. $T_{i}$ is the deadline given by the grid application $i$ to complete its all jobs. $y_{i}^{k}$ is the network allocation obtained by grid application $i$ from the network resource $k . x_{i}^{j}$ is the computing power obtained by grid application $i$ from computing power $j$.

We assume that the mobile grid has heterogeneous nodes with different system performance rates and network conditions. This means that the energy consumption of the mobile device can vary with the response time of the application and the network bandwidth. We denote $e_{i}^{l}$ is the consumed energy fraction of the energy $l$ (e.g. a battery) by grid application $i$. Total consumed energy of all grid applications $\sum_{i=1}^{I} e_{i}^{l}$ does not exceed the total capacity $C e_{l}$ of energy $l$. Energy consumption rate of each node in the system is measured by Joule per unit time. Let $e_{i}^{n}$ be an energy dissipation caused by grid application $i$ ' $\mathrm{s} n$th job, $t_{i}^{n}$ is the execution time of job $n$ of grid application $i$ on the grid node. er is the energy consumption rate of energy resource $l$. If the energy consumption is proportional to execution time of job $n$, as is the case with battery energy. We define the energy consumption of each application $A_{i}$ as the sum of the energy consumed by $N$ grid jobs $\sum_{n=1}^{N} e_{i}^{n}$. The energy consumption of all grid jobs of each application $A_{i}$ should be less than the available resources of $e_{i}^{l}$ which is limited energy budget of grid user application $i$.

Now, we formulate the problem of energy efficient resource management in mobile grid as constraint optimization problem, the utility of the mobile grid $U_{\text {mobilegrid }}$ is defined as the sum of grid application utilities $\sum_{i=1}^{I} U_{i}$. The objective of energy efficient resource management in mobile grid is to maximize the utility of the mobile grid $U_{\text {mobilegrid }}$. By using nonlinear optimization theory, energy efficient resource management in mobile grid can be formulated as follows.

$$
\begin{aligned}
& \text { Max } U_{\text {mobilegrid }} \\
& \text { s.t. } T_{i} \geqslant \sum_{n=1}^{N} t_{i}^{n}, \mathrm{C} n_{k} \geqslant \sum_{i=1}^{I} y_{i}^{k}, \sum_{n=1}^{N} e_{i}^{n} \leqslant e_{i}^{l}, \mathrm{Cc}_{j} \geqslant \sum_{i=1}^{I} x_{i}^{j}, \sum_{i=1}^{I} e_{i}^{l} \leqslant C e_{l} \\
& B_{i} \geqslant \sum_{l=1}^{L} P e_{i}^{l}+\sum_{j=1}^{J} P c_{i}^{j}+\sum_{k=1}^{K} P n_{i}^{k}
\end{aligned}
$$


Where $e_{i}^{l}$ is the energy obtained by grid application $i$ from the energy $l . x_{i}^{j}$ is CPU allocation obtained by grid application $i$ from the computing resource provider $j . y_{i}^{k}$ is bandwidth allocation obtained by grid application $i$ from the network resource provider $k$. The utility function for application $A_{i}$ depends on allocated resources $x_{i}^{j}, y_{i}^{k}$ and consumed energy $e_{i}^{l}$. In the problem Eq. (1), the first type of constraints is related with different resource capacity. The QoS constraint implies that the aggregate network resource units $\sum_{i=1}^{I} y_{i}^{k}$ do not exceed the total capacity $C n_{k}$ of network resource provider $k$, aggregate consumed energy of all grid application $\sum_{i=1}^{I} e_{i}^{l}$ does not exceed the total $C e_{l}$ of energy $l$, aggregate computing power $\sum_{i=1}^{I} x_{i}^{j}$ does not exceed the total resource $C c_{j}$ of the computing resource provider $j$. The second type of constraints is related with grid application expense budget. Grid application needs to complete a sequence of jobs in a specified amount of time, $T_{i}$, while the payment overhead accrued cannot exceed $B_{i}$, which is the expense budget of grid application $i . P e_{i}^{l}, P c_{i}^{j}, P n_{i}^{k}$ are the payments of the grid application $i$ to the energy storage provider $l$, computing resource provider $j$ and network resource provider $k$. The total payments of the grid application

$$
i \sum_{l=1}^{L} P e_{i}^{l}+\sum_{j=1}^{J} P c_{i}^{j}+\sum_{k=1}^{K} P n_{i}^{k}
$$

dose not exceed $B_{i}$. The total energy consumed by all jobs of grid application $i \sum_{n=1}^{N} e_{i}^{n}$ cannot exceed the energy budget $e_{i}^{l}$ which is the available energy obtained by grid application $i$ from the energy storage $l$.

\subsection{Solutions to mathematic model}

We apply the Lagrangian method to solve such a problem Eq. (1). The Lagrangian approach can be used to solve constrained optimization problems. Let us consider the Lagrangian form of energy efficient resource management in mobile grid:

$$
\begin{aligned}
L & =\sum_{i=1}^{I} U_{i}-\lambda_{i}\left(\sum_{i=1}^{I} e_{i}^{l}-C e_{l}\right)-\beta_{i}\left(\sum_{i=1}^{I} x_{i}^{j}-C c_{j}\right)-\varphi_{i}\left(\sum_{i=1}^{I} y_{i}^{k}-C n_{k}\right) \\
& -\gamma_{i}\left(\sum_{l=1}^{L} P e_{i}^{l}+\sum_{j=1}^{J} P c_{i}^{j}+\sum_{k=1}^{K} P n_{i}^{k}-B_{i}\right)-\mu_{i}\left(\sum_{n=1}^{N} e_{i}^{n}-e_{i}^{l}\right)-\alpha_{i}\left(\sum_{n=1}^{N} t_{i}^{n}-T_{i}\right)
\end{aligned}
$$

where $\lambda_{i}, \beta_{i}$ and $\varphi_{i}$ are the Lagrange multipliers of grid application with their interpretation of energy price, computing resource capacity price, and network resource capacity price, respectively. Since the Lagrangian is separable, this maximization of Lagrangian over $\left(x_{i}^{j}, y_{i}^{k}, e_{i}^{l}\right)$ can be conducted in parallel at each application $A_{i}$. In problem Eq. (1), though the allocated resources $x_{i}^{j}, y_{i}^{k}$ and consumed energy $e_{i}^{l}$ are coupled in their constraints, respectively, but they are separable. Given that the grid knows the utility functions $U$ of all the grid applications, this optimization problem can be mathematically tractable. However, in practice, it is not likely to know each application's utility, and it is also infeasible for mobile grid environment to compute and allocate resources in a centralized fashion. In order to derive a distributed algorithm to solve problem Eq. (1), we decompose the problem into the sub problems. 
The mobile grid utility denoted as the sum of grid application utility can be defined as follows Eq. (2):

$$
\begin{array}{r}
U_{\text {mobilegrid }}=\left(B_{i}-\sum_{l=1}^{L} P e_{i}^{l}-\sum_{j=1}^{J} P c_{i}^{j}-\sum_{k=1}^{K} P n_{i}^{k}\right)+\left(T_{i}-\sum_{n=1}^{N} t_{i}^{n}\right) \\
+\sum_{i=1}^{I}\left(P e_{i}^{l} \log e_{i}^{l}+P c_{i}^{j} \log x_{i}^{j}+P n_{i}^{k} \log y_{i}^{k}\right)+\left(e_{i}^{l}-\sum_{n=1}^{N} e_{i}^{n}\right)
\end{array}
$$

Mobile grid utility function is maximally optimized with specific constraints. In Eq. (2), $P e_{i}^{l} \log e_{i}^{l}+$ $P c_{i}^{j} \log x_{i}^{j}+P n_{i}^{k} \log y_{i}^{k}$ present the revenue of energy storage resource, computing power and network resource provider. We could have chosen any other form for the utility that increases with $x_{i}^{j}, y_{i}^{k}, e_{i}^{l}$. But we chose the $\log$ function because the benefit increases quickly from zero as the total allocated resource increases from zero and then increases slowly. Moreover, $\log$ function is analytically convenient, increasing, strictly concave and continuously differentiable. The benefits of grid resource provider are affected by payments of grid applications and allocated resources. It means that the revenue increases with increasing allocated resources and increasing payment.

The Lagrangian form of the problem Eq. (1) can be reformulated as follows Eq. (3):

$$
\begin{aligned}
L & =\left(B_{i}-\sum_{l=1}^{L} P e_{i}^{l}-\sum_{j=1}^{J} P c_{i}^{j}-\sum_{k=1}^{K} P n_{i}^{k}\right)+\sum_{i=1}^{I}\left(P e_{i}^{l} \log e_{i}^{l}+P c_{i}^{j} \log x_{i}^{j}+P n_{i}^{k} \log y_{i}^{k}\right) \\
& +\left(T_{i}-\sum_{n=1}^{N} t_{i}^{n}\right)+\left(e_{i}^{l}-\sum_{n=1}^{N} e_{i}^{n}\right)-\lambda_{i}\left(\sum_{i=1}^{I} e_{i}^{l}-C e_{l}\right)-\beta_{i}\left(\sum_{i=1}^{I} x_{i}^{j}-C c_{j}\right) \\
& -\varphi_{i}\left(\sum_{i=1}^{I} y_{i}^{k}-C n_{k}\right)-\gamma_{i}\left(\sum_{l=1}^{L} P e_{i}^{l}+\sum_{j=1}^{J} P c_{i}^{j}+\sum_{k=1}^{K} P n_{i}^{k}-B_{i}\right)-\mu_{i}\left(\sum_{n=1}^{N} e_{i}^{n}-e_{i}^{l}\right) \\
& -\alpha_{i}\left(\sum_{n=1}^{N} t_{i}^{n}-T_{i}\right)
\end{aligned}
$$

The system model presented by Eq. (1) is a nonlinear optimization problem with $N$ decision variables. Since the Lagrangian is separable, the maximization of the Lagrangian can be processed in parallel for grid user applications and grid resource providers respectively. From Eq. (3), the resource allocation $\left\{e_{i}^{l}\right.$, $\left.x_{i}^{j}, y_{i}^{k}\right\}$ solves problem Eq. (1) if and only if there exist a set of nonnegative shadow costs $\left\{\lambda_{i}, \beta_{i}, \varphi_{i}\right\}$. Generally solving such a problem by typical algorithms such as steepest decent method and gradient projection method is of high computational complexity, which is very time costing and impractical for implementation. In order to reduce the computational complexity, we decompose the utility optimization problem Eq. (1) into two subproblems for grid user applications and grid resource providers so that the computational complexity is reduced. The shadow costs suggest a mechanism to distribute the resource optimization between the grid applications and the grid system. The problem Eq. (1) maximizes the utility of grid applications on the energy price, computing power capacity price, and network resource capacity price, $\sum_{i=1}^{I} U_{i}\left(e_{i}^{l}, x_{i}^{j}, y_{i}^{k}\right)$ is the total utility of mobile grid system, $\beta_{i} \sum_{i=1}^{I} x_{i}^{j}$ is the computing power cost, $\lambda_{i} \sum_{i=1}^{I} e_{i}^{l}$ is the energy cost, $\varphi_{i} \sum_{i=1}^{I} y_{i}^{k}$ is the network resource cost. By decomposing 
the Kuhn-Tucker conditions into separate roles of consumer and supplier at grid market, the centralized problem Eq. (1) can be transformed into a distributed problem. Grid application's payment is collected by the resource providers. The payments of grid applications paid to resource providers are the payments to resolve the optimality of resource allocation in the grid market. We decompose the problem into the following two subproblems Eq. (4) which is grid users optimization problem and Eq. (5) which is grid resource providers optimization problem, seek a distributed solution where the grid resource provider does not need to know the utility functions of individual grid user application. Equations (4), (5) derived from the distributed approach are identical to the optimal conditions given by centralized energy efficient resource management problem Eq. (1). This means if two subproblems converge to its optimal points, then a globally optimal point is achieved. Total user application benefit of the mobile grid is maximized when the equilibrium prices, obtained through the two subproblems Eqs (4) and (5), equal the Lagrangian multipliers $\lambda_{i}, \beta_{i}$ and $\varphi_{i}$, where $\lambda_{i}, \beta_{i}$ and $\varphi_{i}$ are the optimal prices charged by resource providers including energy, computing power and network resource to grid applications. Two maximization subproblems correspond to grid application optimization problem as denoted by Eqs (4) and (5).

$$
\begin{aligned}
\operatorname{Max}_{\mathrm{GA}}= & \left(T_{i}-\sum_{n=1}^{N} t_{i}^{n}\right)+\left(e_{i}^{l}-\sum_{n=1}^{N} e_{i}^{n}\right)+\left(B_{i}-\sum_{l=1}^{L} P e_{i}^{l}-\sum_{j=1}^{J} P c_{i}^{j}-\sum_{k=1}^{K} P n_{i}^{k}\right) \\
B_{i} \geqslant & \sum_{l=1}^{L} P e_{i}^{l}+\sum_{j=1}^{J} P c_{i}^{j}+\sum_{k=1}^{K} P n_{i}^{k} \\
& \sum_{n=1}^{N} e_{i}^{n} \leqslant e_{i}^{l}, T_{i} \geqslant \sum_{n=1}^{N} t_{i}^{n}, \\
\operatorname{MaxU}_{\mathrm{GR}}= & \sum_{i=1}^{I}\left(P e_{i}^{l} \log e_{i}^{l}+P c_{i}^{j} \log x_{i}^{j}+P n_{i}^{k} \log y_{i}^{k}\right) \\
& \mathrm{Cc}_{j} \geqslant \sum_{i=1}^{I} x_{i}^{j}, \quad \mathrm{C}_{k} \geqslant \sum_{i=1}^{I} y_{i}^{k}, \quad \sum_{i=1}^{I} e_{i}^{l} \leqslant C e_{l}
\end{aligned}
$$

In Eq. (4), the grid application gives the unique optimal payment to resource provider under the energy budget, expense budget and the deadline constraint to maximize the user's satisfaction.

$$
\left(B_{i}-\sum_{l=1}^{L} P e_{i}^{l}-\sum_{j=1}^{J} P c_{i}^{j}-\sum_{k=1}^{K} P n_{i}^{k}\right)
$$

represents the money surplus of grid application $i$, which is obtained by expense budgets subtracting the payments to various resource providers. $\left(T_{i}-\sum_{n=1}^{N} t_{i}^{n}\right)$ represents the saving times for grid application $i$, which is gotten by time limit subtracting actual spending time. $\left(e_{i}^{l}-\sum_{n} e_{i}^{n}\right)$ represents the energy surplus of grid application $i$ which is obtained by the energy budgets subtracting actual energy dissipation. So, the objective of Eq. (4) is to get more surpluses of money and more energy savings, at the same time, complete task for grid user application as soon as possible. In Eq. (5), different resource providers 
compute optimal resource allocation for maximizing the revenue of their own. Grid application $i$ submits the payment $P e_{i}^{l}$ to the energy resource provider $l, P n_{i}^{k}$ to network resource provider $k$ and $P c_{i}^{j}$ to computing power provider $j . P e_{i}^{l} \log e_{i}^{l}$ presents the revenue obtained by energy resource $l$ from grid application $i . P c_{i}^{j} \log x_{i}^{j}$ presents the revenue obtained by computing power $j$ from grid application $i$. $P n_{i}^{k} \log y_{i}^{k}$ presents the revenue obtained by network resource $k$ from grid application $i$. The objective of resource providers is to maximize

$$
P e_{i}^{l} \log e_{i}^{l}+P c_{i}^{j} \log x_{i}^{j}+P n_{i}^{k} \log y_{i}^{k}
$$

under the constraints of their provided resource amounts. Grid resource providers can't sell the resources to grid applications more than total capacity. In Eq. (4), the grid application adaptively adjusts its payments to computing power, network resource and energy based on the current resource conditions, while in Eq. (5), the grid resource provider adaptively allocates energy, CPU and bandwidth required by the grid application in the Eq. (4). The interaction between two sub-problems is controlled through the use of the price variable $\lambda_{i}, \beta_{i}$ and $\varphi_{i}$, which is the energy price, computing power price, and network resource price charged from grid applications by grid energy resource, computing power and network resource. The interaction between two sub-problems also coordinates the grid application's payment and the supply of grid resource providers.

Lagrange relaxation and gradient optimization can be applied to decompose such an overall optimization problem into a sequence of two sub-problems, each only involving variables from the grid application and resource providers respectively. In Eq. (4), grid application maximizes its satisfaction and gives the unique optimal payment to resource provider under the energy budget, expense budget and the deadline constraint. We assume that grid application $i$ submits $P e_{i}^{l}$ to energy resource $l, P c_{i}^{j}$ to computing power $j$ and $P n_{i}^{k}$ to network resource $k$. Then, $P e_{i}=\left[P e_{i}^{1}, \ldots, P e_{i}^{l}\right]$ represents all payments of grid applications for energy resource $l, P c_{i}=\left[P c_{i}^{1}, \ldots, P c_{i}^{j}\right]$ represents all payments of grid applications for computing power $j, P n_{i}=\left[P n_{i}^{1}, \ldots, P n_{i}^{k}\right]$ represents all payments of grid applications for the network resource $k$. Let

$$
m_{i}=\sum_{l} P e_{i}^{l}+\sum_{j} P c_{i}^{j}+\sum_{k} P n_{i}^{k}
$$

$m_{i}$ is the total payment of the $i$ th grid application. $N$ grid applications compete for grid resources with finite capacity. The resource is allocated using a market mechanism, where the partitions depend on the relative payments sent by the grid applications. Let $e p_{l}, c p_{j}, n p_{k}$ denote the price of the resource unit of energy resource $l$, the price of the resource unit of computing power $j$ and network resource $k$ respectively. Let the pricing policy, $e p=\left(e p_{1}, e p_{2}, \cdots, e p_{l}\right)$, denote the set of resource unit prices of all the energy resources in the grid, $c p=\left(c p_{1}, c p_{2}, \cdots, c p_{j}\right)$, denote the set of resource unit prices of all the computing powers, $n p=\left(n p_{1}, n p_{2}, \cdots, n p_{k}\right)$ is set of network resource unit prices. The $i$ th grid application receives the resources proportional to its payment relative to the sum of the resource provider's revenue. Let $e_{i}^{l}, i^{j}, y_{i}^{k}$ be the fraction of resource units allocated to grid application $i$ by energy $l$, computing power $j$ and network resource $k$.

The time taken by the $i$ th grid application to complete $n$th job is:

$$
t_{i}^{n}=\frac{c q_{i}^{n} c p_{j}}{C c_{j} P c_{i}^{j}}+\frac{b q_{i}^{n} n p_{k}}{C n_{k} P n_{i}^{k}}+\frac{e q_{i}^{n} e p_{l}}{C e_{l} P e_{i}^{l}}
$$


The energy dissipation used by the $i$ th grid user to complete $n$th job is:

$$
e_{i}^{n}=e r . t_{i}^{n}=e r \cdot\left(\frac{c q_{i}^{n} c p_{j}}{C c_{j} P c_{i}^{j}}+\frac{b q_{i}^{n} n p_{k}}{C n_{k} P n_{i}^{k}}+\frac{e q_{i}^{n} e p_{l}}{C e_{l} P e_{i}^{l}}\right)
$$

Grid user optimization can be reformulated as

$$
\begin{aligned}
\operatorname{Max}_{\mathrm{GA}} & =\left(B_{i}-\sum_{l=1}^{L} P e_{i}^{l}-\sum_{j=1}^{J} P c_{i}^{j}-\sum_{k=1}^{K} P n_{i}^{k}\right)+\left(T_{i}-\sum_{n=1}^{N}\left(\frac{c q_{i}^{n} c p_{j}}{C c_{j} P c_{i}^{j}}+\frac{b q_{i}^{n} n p_{k}}{C n_{k} P n_{i}^{k}}+\frac{e q_{i}^{n} e p_{l}}{C e_{l} P e_{i}^{l}}\right)\right) \\
& +\left(e_{i}^{l}-\sum_{n=1}^{N} \operatorname{er}\left(\frac{c q_{i}^{n} c p_{j}}{C c_{j} P c_{i}^{j}}+\frac{b q_{i}^{n} n p_{k}}{C n_{k} P n_{i}^{k}}+\frac{e q_{i}^{n} e p_{l}}{C e_{l} P e_{i}^{l}}\right)\right)
\end{aligned}
$$

The Lagrangian for the grid application's utility is $L_{1}\left(P e_{i}^{l}, P c_{i}^{j}, P n_{i}^{k}\right)$.

$$
\begin{aligned}
L_{1}\left(P e_{i}^{l}, P c_{i}^{j},\right. & \left.P n_{i}^{k}\right)=\left(B_{i}-\sum_{l=1}^{L} P e_{i}^{l}-\sum_{j=1}^{J} P c_{i}^{j}-\sum_{k=1}^{K} P n_{i}^{k}\right) \\
& +\left(T_{i}-\sum_{n=1}^{N}\left(\frac{c q_{i}^{n} c p_{j}}{C c_{j} P c_{i}^{j}}+\frac{b q_{i}^{n} n p_{k}}{C n_{k} P n_{i}^{k}}+\frac{e q_{i}^{n} e p_{l}}{C e_{l} P e_{i}^{l}}\right)\right) \\
& +\left(e_{i}^{l}-\sum_{n=1}^{N} e r\left(\frac{c q_{i}^{n} c p_{j}}{C c_{j} P c_{i}^{j}}+\frac{b q_{i}^{n} n p_{k}}{C n_{k} P n_{i}^{k}}+\frac{e q_{i}^{n} e p_{l}}{C e_{l} P e_{i}^{l}}\right)\right) \\
& +\nu_{i}\left(B_{i}-\sum_{l=1}^{L} P e_{i}^{l}-\sum_{j=1}^{J} P c_{i}^{j}-\sum_{k=1}^{K} P n_{i}^{k}\right) \\
& +\sigma_{i}\left(T_{i}-\sum_{n=1}^{N}\left(\frac{c q_{i}^{n} c p_{j}}{C c_{j} P c_{i}^{j}}+\frac{b q_{i}^{n} n p_{k}}{C n_{k} P n_{i}^{k}}+\frac{e q_{i}^{n} e p_{l}}{C e_{l} P e_{i}^{l}}\right)\right) \\
& +\varepsilon_{i}\left(e_{i}^{l}-\sum_{n=1}^{N} e r\left(\frac{c q_{i}^{n} c p_{j}}{C c_{j} P c_{i}^{j}}+\frac{b q_{i}^{n} n p_{k}}{C n_{k} P n_{i}^{k}}+\frac{e q_{i}^{n} e p_{l}}{C e_{l} P e_{i}^{l}}\right)\right)
\end{aligned}
$$

Where $\varepsilon_{i}, \sigma_{i}, \nu_{i}$ is the Lagrangian constant. From Karush-Kuhn-Tucker Theorem we know that the optimal solution is given $\partial L_{1}\left(P e_{i}^{l}, P c_{i}^{j}, P n_{i}^{k}\right) / \partial P e_{i}^{l}=0$ for $\varepsilon_{i}, \sigma_{i}, \nu_{i}>0$.

$$
\begin{array}{r}
\partial L_{1}\left(P e_{i}^{l}, P c_{i}^{j}, P n_{i}^{k}\right) / \partial P e_{i}^{l}=-1- \\
-\nu_{i}+\frac{e q_{i}^{n} e p_{l}}{C e_{l}\left(P e_{i}^{l}\right)^{2}}+e r \frac{e q_{i}^{n} e p_{l}}{C e_{l}\left(P e_{i}^{l}\right)^{2}} \\
+\sigma_{i} \frac{e q_{i}^{n} e p_{l}}{C e_{l}\left(P e_{i}^{l}\right)^{2}}+\varepsilon_{i} \cdot e r \frac{e q_{i}^{n} e p_{l}}{C e_{l}\left(P e_{i}^{l}\right)^{2}}
\end{array}
$$


Let $\partial L_{1}\left(P e_{i}^{l}, P c_{i}^{j}, P n_{i}^{k}\right) / \partial P e_{i}^{l}=0$ to obtain

$$
P e_{i}^{l}=\left(\frac{\left(1+e r+\sigma_{i}+\varepsilon_{i} \cdot e r\right) e q_{i}^{n} e p_{l}}{\left(1+\nu_{i}\right) C e_{l}}\right)^{1 / 2}
$$

Using this result in the constraint equation, we can determine $\theta=\left(1+e r+\sigma_{i}+\varepsilon_{i} \cdot e r\right) / 1+\nu_{i}$ as

$$
(\theta)^{-1 / 2}=\frac{T_{i}}{\sum_{m=1}^{N}\left(\frac{e p_{m} e q_{i}^{n}}{C e_{m}}\right)^{1 / 2}}
$$

We obtain $P e_{i}^{l *}$

$$
P e_{i}^{l^{*}}=\left(\frac{e q_{i}^{n} e p_{l}}{C e_{l}}\right)^{1 / 2} \frac{\sum_{m=1}^{N}\left(\frac{e q_{i}^{n} e p_{m}}{C e_{m}}\right)^{1 / 2}}{T_{i}}
$$

It means that grid application wants to pay $P e_{i}^{l^{*}}$ to energy resource $l$ for needed energy consumed to execute grid jobs under completion time constraint.

$$
\begin{aligned}
\partial L_{1}\left(P e_{i}^{l}, P c_{i}^{j}, P n_{i}^{k}\right) / \partial P c_{i}^{j}= & -1+\frac{c q_{i}^{n} c p_{j}}{C c_{j}\left(P c_{i}^{j}\right)^{2}}+e r_{i}^{n} \frac{c q_{i}^{n} c p_{j}}{C c_{j}\left(P c_{i}^{j}\right)^{2}}-\nu_{i} \\
& +\sigma_{i} \frac{c q_{i}^{n} c p_{j}}{C c_{j}\left(P c_{i}^{j}\right)^{2}}+\varepsilon_{i} \cdot e r \frac{c q_{i}^{n} c p_{j}}{C c_{j}\left(P c_{i}^{j}\right)^{2}}
\end{aligned}
$$

Let $\partial L_{1}\left(P e_{i}^{l}, P c_{i}^{j}, P n_{i}^{k}\right) / \partial P c_{i}^{j}=0$ to obtain

$$
P c_{i}^{j}=\left(\frac{\left(1+e r+\sigma_{i}+\varepsilon_{i} . e r\right) c q_{i}^{n} c p_{j}}{\left(1+\nu_{i}\right) C c_{j}}\right)^{1 / 2}
$$

Using this result in the constraint equation, we can determine $\xi=\left(1+e r+\sigma_{i}+\varepsilon_{i} \cdot e r\right) / 1+\nu_{i}$ as

$$
(\xi)^{-1 / 2}=\frac{T_{i}}{\sum_{m=1}^{N}\left(\frac{c p_{m} c q_{i}^{n}}{C c_{m}}\right)^{1 / 2}}
$$

We obtain $P c_{i}^{j^{*}}$

$$
P c_{i}^{j^{*}}=\left(\frac{c q_{i}^{n} c p_{j}}{C c_{j}}\right)^{1 / 2} \frac{\sum_{m=1}^{N}\left(\frac{c q_{i}^{n} c p_{m}}{C c_{m}}\right)^{1 / 2}}{T_{i}}
$$

It means that grid application wants to pay $P c_{i}^{j^{*}}$ to computing power $j$ for needed resource to execute grid jobs under completion time constraint.

$$
\partial L_{1}\left(P e_{i}^{l}, P c_{i}^{j}, P n_{i}^{k}\right) / \partial P n_{i}^{k}=-1+\frac{b q_{i}^{n} n p_{k}}{C n_{k}\left(P n_{i}^{k}\right)^{2}}+e r_{i}^{n} \frac{b q_{i}^{n} n p_{k}}{C n_{k}\left(P n_{i}^{k}\right)^{2}}-\nu_{i}
$$




$$
+\sigma_{i} \frac{b q_{i}^{n} n p_{k}}{C n_{k}\left(P n_{i}^{k}\right)^{2}}+\varepsilon_{i} \frac{b q_{i}^{n} n p_{k}}{C n_{k}\left(P n_{i}^{k}\right)^{2}}
$$

Let $\partial L_{1}\left(P e_{i}^{l}, P c_{i}^{j}, P n_{i}^{k}\right) / \partial P n_{i}^{k}=0$ to obtain

$$
P n_{i}^{k}=\left(\frac{\left(1+e r+\sigma_{i}+e r \cdot \varepsilon_{i}\right) b q_{i}^{n} n p_{k}}{\left(1+\nu_{i}\right) C n_{k}}\right)^{1 / 2}
$$

Using this result in the constraint equation, we can determine $\tau=\left(1+e r+\sigma_{i}+e r \cdot \varepsilon_{i}\right) / 1+\nu_{i}$ as

$$
(\tau)^{-1 / 2}=\frac{T_{i}}{\sum_{m=1}^{N}\left(\frac{n p_{m} b q_{i}^{n}}{C n_{m}}\right)^{1 / 2}}
$$

We obtain $P n_{i}^{k^{*}}$

$$
P n_{i}^{k^{*}}=\left(\frac{b q_{i}^{n} n p_{k}}{C n_{k}}\right)^{1 / 2} \frac{\sum_{m=1}^{N}\left(\frac{b q_{i}^{n} n p_{m}}{C n_{m}}\right)^{1 / 2}}{T_{i}}
$$

It means that grid application wants to pay $P n_{i}^{k^{*}}$ to network resource $k$ for needed resource to execute grid jobs under completion time constraint.

In Eq. (5), different resource providers compute optimal resource allocation for maximizing the revenue of their own under constrains of resource capacity $C e_{l}, \mathrm{Cc}_{j}, \mathrm{C} n_{k}$, the objective of resource providers is to maximize

$$
P e_{i}^{l} \log e_{i}^{l}+P c_{i}^{j} \log x_{i}^{j}+P n_{i}^{k} \log y_{i}^{k}
$$

under the constraints of their resource capacity.

We take first derivative and second derivative with respect to $x_{i}$ :

$$
U_{\mathrm{GR}}^{\prime}\left(e_{i}^{l}\right)=P e_{i}^{l} / e_{i}^{j} \quad U_{\mathrm{GR}}^{\prime \prime}\left(e_{i}^{l}\right)=-P e_{i}^{l} / e_{i}^{l 2}
$$

$U_{\mathrm{GR}}^{\prime \prime}\left(e_{i}^{l}\right)<0$ is negative due to $0<e_{i}^{l}$. The extreme point is the unique value maximizing the revenue of energy provider. The Lagrangian for (3.8) is $L_{2}\left(e_{i}^{l}, x_{i}^{j}, y_{i}^{k}\right)$.

$$
\begin{aligned}
\mathrm{L}_{2}\left(e_{i}^{l}, x_{i}^{j}, y_{i}^{k}\right)= & \sum\left(P e_{i}^{l} \log e_{i}^{l}+P c_{i}^{j} \log x_{i}^{j}+P n_{i}^{k} \log y_{i}^{k}\right) \\
& +\lambda_{i}\left(C e_{l}-\sum_{i} e_{i}^{l}\right)+\beta_{i}\left(C c_{j}-\sum_{i} x_{i}^{j}\right)+\varphi_{i}\left(C n_{k}-\sum_{i} y_{i}^{k}\right) \\
& =\sum\left(P e_{i}^{l} \log e_{i}^{l}+P c_{i}^{j} \log x_{i}^{j}+P n_{i}^{k} \log y_{i}^{k}-\lambda_{i} e_{i}^{l}-\beta_{i} x_{i}^{j}-\varphi_{i} y_{i}^{k}\right) \\
& +\lambda_{i} C e_{l}+\beta_{i} C c_{j}+\varphi_{i} C n_{k}
\end{aligned}
$$


Where $\lambda_{i}, \beta_{i}$ and $\varphi_{i}$, is the Lagrangian constant. From Karush-Kuhn-Tucker Theorem we know that the optimal solution is given $\partial L_{2}\left(e_{i}^{l}, x_{i}^{j}, y_{i}^{k}\right) / \partial e_{i}^{l}=0$ for $\lambda_{i}, \beta_{i}, \varphi_{i}>0$.

Let $\partial L_{2}\left(e_{i}^{l}, x_{i}^{j}, y_{i}^{j}\right) / \partial e_{i}^{l}=0$ to obtain $\quad e_{i}^{l}=P e_{i}^{l} / \lambda_{i}$

Using this result in the constraint equation $C e_{l} \geqslant \sum e_{i}^{l}$, we can determine $\lambda_{i}$ as

$$
\lambda_{i}=\frac{\sum_{d=1}^{n} P e_{i}^{d}}{C e_{l}}
$$

We substitute $\lambda$ into $e_{i}^{l}$ to obtain

$$
e_{i}^{l^{*}}=\frac{P e_{i}^{l} C e_{l}}{\sum_{d=1}^{n} P e_{i}^{d}}
$$

$e_{i}^{l^{*}}$ is the unique energy allocation for maximizing the revenue of energy provider $l$.

Using the similar method, we can solve computing power allocation optimization problem.

Let $\partial L_{2}\left(e_{i}^{l}, x_{i}^{j}, y_{i}^{k}\right) / \partial x_{i}^{j}=0$ to obtain $x_{i}^{j}=P c_{i}^{j} / \beta_{i}$

Using this result in the constraint equation $C c_{j} \geqslant \sum x_{i}^{j}$, we can determine $\beta_{i}$ as

$$
\beta_{i}=\frac{\sum_{d=1}^{n} P c_{i}^{d}}{C c_{j}}
$$

We substitute $\beta$ into $x_{i}^{j}$ to obtain

$$
x_{i}^{j^{*}}=\frac{P c_{i}^{j} C c_{j}}{\sum_{d=1}^{n} P c_{i}^{d}}
$$

$x_{i}^{j^{*}}$ is the unique optimal computing power allocation for maximizing the revenue of computing power provider $j$.

Using the similar method, we can solve network resource allocation optimization problem.

Let $\partial L_{2}\left(e_{i}^{l}, x_{i}^{j}, y_{i}^{k}\right) / \partial y_{i}^{k}=0$ to obtain $y_{i}^{k}=P n_{i}^{k} / \varphi_{i}$

Using this result in the constraint equation $C n_{k} \geqslant \sum y_{i}^{k}$, we can determine $\varphi_{i}$ as

$$
\varphi_{i}=\frac{\sum_{d=1}^{n} P n_{i}^{d}}{C n_{k}}
$$

We substitute $\varphi$ into $y_{i}^{k}$ to obtain

$$
y_{i}^{k^{*}}=\frac{P n_{i}^{k} C n_{k}}{\sum_{d=1}^{n} P n_{i}^{d}}
$$


$y_{i}^{k^{*}}$ is the unique optimal network resource allocation for maximizing the revenue of network resource provider $k$.

\section{Energy efficient resource management algorithm in mobile grid}

Energy efficient resource management algorithm decomposes energy consumption optimization problem into a sequence of sub-problems via an iterative algorithm. In each iteration, in the routine of grid user optimization, the grid application computes the unique optimal payment to resource provider under the energy budget, expense budget and the deadline constraint to maximize the grid application's satisfaction. The grid application individually solves its fees to pay for energy resources, computing power and network resource to complete its all jobs, adjusts its grid resource demand and notifies the grid resource provider about this change. In the routine of grid resource provider optimization, different resource providers compute optimal resource allocation for maximizing the revenue of their own. Grid resource provider updates its price according to optimal payments from grid application, and then sends the new prices to the grid applications and allocates the resource for grid application, and the cycle

repeats. The algorithm that achieves energy efficient resource management in mobile grid is described as follows.

\section{Algorithm 1 Energy Efficient Resource Management Algorithm (EERM)}

\section{Sub-algorithm 1}

Step 1: Receives the new price $e p_{l}$ from the energy provider $l$;

Step 2: $P e_{i}^{l^{*}}=\operatorname{Max}\left\{U_{a p p}\left(P e_{i}^{l}, P c_{i}^{j}, P n_{i}^{k}\right)\right\}$;

Step 3: If $B_{i} \geqslant \sum_{j} P c_{i}^{j}+\sum_{k} P n_{i}^{k}+\sum_{l} P e_{i}^{l}$

Then Return $P e_{i}^{l^{*}}$ to energy resource $l$;

Step 4: Else Return Null;

Step 5: Receives the new price $c p_{j}$ from the computing power $j$;

Step 6: $P c_{i}^{j^{*}}=\operatorname{Max}\left\{U_{\text {app }}\left(P e_{i}^{l}, P c_{i}^{j}, P n_{i}^{k}\right)\right\}$;

Step 7: If $B_{i} \geqslant \sum_{j} P c_{i}^{j}+\sum_{k} P n_{i}^{k}+\sum_{l} P e_{i}^{l}$

Then Return $P c_{i}^{j^{*}}$ to computing power $j$;

Step 8: Else Return Null;

Step 9: Receives the new price $n p_{k}$ from the network resource provider $k$;

Step 10: $P n_{i}^{k^{*}}=\operatorname{Max}\left\{U_{a p p}\left(P e_{i}^{l}, P c_{i}^{j}, P n_{i}^{k}\right)\right\}$;

Step 11: If $B_{i} \geqslant \sum_{j} P c_{i}^{j}+\sum_{k} P n_{i}^{k}+\sum_{l} P e_{i}^{l}$

Then Return $P n_{i}^{k^{*}}$ to network resource $k$;

Step 12: Else Return Null;

Sub-algorithm 2

Step 1: Receives optimal payments $P e_{i}^{l^{*}}, P c_{i}^{j^{*}}, P n_{i}^{k^{*}}$ from grid application $i$; 
Step 2: If $C e_{l} \geqslant \sum_{i=1}^{I} e_{i}^{l}$

Then

$$
\begin{aligned}
& e_{i}^{l^{(n+1) *}}=\operatorname{Max}\left\{U_{\text {resource }}\left(e_{i}^{l}, x_{i}^{j}, y_{i}^{k}\right)=\sum_{i=1}^{I}\left(P e_{i}^{l} \log e_{i}^{l}+P c_{i}^{j} \log x_{i}^{j}+P n_{i}^{k} \log y_{i}^{k}\right)\right\} ; \\
& e p_{l}^{(n+1)}=\max \left\{\varepsilon, e p_{l}^{(n)}+\eta\left(e^{l} e p_{l}^{(n)}-C e_{l}\right)\right\} ;
\end{aligned}
$$

Return energy resource price $e p_{l}^{(n+1)}$ to all grid applications;

Step 3: Else Return Null;

Step 4: If $\mathrm{C} c_{i} \geqslant \sum_{i=1}^{I} x_{i}^{j}$

Then

$$
x_{i}^{j^{(n+1) *}}=\operatorname{Max}\left\{U_{\text {resource }}\left(e_{i}^{l}, x_{i}^{j}, y_{i}^{k}\right)=\sum_{i=1}^{I}\left(P e_{i}^{l} \log e_{i}^{l}+P c_{i}^{j} \log x_{i}^{j}+P n_{i}^{k} \log y_{i}^{k}\right)\right\} ;
$$

// Calculates its optimal computing power $x_{i}^{j^{(n+1) *}}$

$c p_{j}^{(n+1)}=\max \left\{\varepsilon, c p_{j}^{(n)}+\eta\left(x^{j} c p_{j}^{(n)}-C c_{j}\right)\right\} ; \quad / /$ Computes a new price

$/ / x^{j}=\sum_{i} x_{i}^{j}, \eta>0$ is a small step size parameter, $n$ is iteration number

Return computing power price $c p_{j}^{(n+1)}$ to all grid applications;

Step 5: Else Return Null;

Step 6: If $C n_{k} \geqslant \sum_{i=1}^{I} y_{i}^{k}$

Then

$$
y_{i}^{k^{(n+1) *}}=\operatorname{Max}\left\{U_{\text {resource }}\left(e_{i}^{l}, x_{i}^{j}, y_{i}^{k}\right)=\sum_{i=1}^{I}\left(P e_{i}^{l} \log e_{i}^{l}+P c_{i}^{j} \log x_{i}^{j}+P n_{i}^{k} \log y_{i}^{k}\right)\right\} ;
$$

// Calculates its optimal network resource demand $y_{i}^{k^{(n+1) *}}$

$n p_{k}^{(n+1)}=\max \left\{\varepsilon, n p_{k}^{(n)}+\eta\left(y^{k} n p_{k}^{(n)}-C n_{k}\right)\right\} ; \quad / /$ Computes a new price

$/ / y^{k}=\sum_{i} y_{i}^{k}, \eta>0$ is a small step size parameter, $n$ is iteration number

Return network resource price $n p_{k}^{(n+1)}$ to all grid applications;

Step 7: Else Return Null;

\section{Simulations}

In this section, we present the performance evaluation of energy efficient resource management algorithm using the JAVASIM [15] simulator. Network generator BRITE [16] generates the computer network topology. We simulate a mobile grid environment with a 2 dimension area of $500 \mathrm{~m} * 500 \mathrm{~m}$ to study mobile device's behavior. Each mobile device in the simulated environment has a maximal radio range of $100 \mathrm{~m}$, and moves following a random-walking mobility model. The average speed of 
each mobile device is 5 meters per second. The average distance between neighboring devices is 25 meters. Mobile devices dynamically enter and leave the mobile grid. There are a number of parameters associated with each device such as energy budget, expense budget, and a two-dimension position value. Each mobile device's battery capacity is initialized with a random value in the range of $[700,800]$, and reduced automatically by a random value in the range of $[0,5]$ in each iteration. There are a total of 150 resources and 600 applications are taken for experimental evaluation of the system. A LAN consists of 90 nodes all of which contribute resources to the grid. The LAN acts as the main Grid infrastructure into which we want to integrate mobile devices. Device schedulers residing in WLANs, acting as the interface point between the mobile devices. All Wi-Fi interfaces operate at a rate of $11 \mathrm{Mb} / \mathrm{s}$. All Ethernet interfaces operate at a rate of $10 \mathrm{~Gb} / \mathrm{s}$. The selective grid applications for simulation are computation-intensive applications such as image processing applications and mpeg players. We assume that each grid application can use any of grid resources including computation, communication and energy resources. Processor capacity varies from 220 to 580 MIPS. The wireless network bandwidth is from $10 \mathrm{Kbps}$ to $1 \mathrm{Mbps}$. The main memory is set by $128 \mathrm{M}, 256 \mathrm{M}, 512 \mathrm{M}$, and $2 \mathrm{G}$. The disk capacity is set by $80 \mathrm{G}, 30 \mathrm{G}, 20 \mathrm{G}$. Energy consumption is represented as a percentage of the total energy required to meet all job deadlines. Assume that the maximum power, $P_{\max }$, corresponds to running all jobs with the maximum processing frequency. The maximum frequency is assumed to be $f_{\max }=1$ and the maximum frequency-dependent power is $P_{\max }=1$. When the energy budget for each interval is limited, we can only consume a fraction of $P_{\max }$ when processing requests during a given interval. Jobs arrive at each site $s_{i}, i=1,2, \ldots, n$ according to a Poisson process with rate $\alpha$. The capacities of the energy resources were chosen uniformly in the interval [50,500]. The energy cost can be expressed in grid dollar that can be defined as unit energy processing cost. The initial price of energy is set from 10 to 500 grid dollars. Users submit their jobs with varying deadlines. The deadlines of grid application are chosen from $100 \mathrm{~ms}$ to $400 \mathrm{~ms}$. The budgets of grid applications are set from 100 to 1500 grid dollars. Each experiment is repeated 6 times and 95\% confidence intervals are obtained. The simulation results shown in the figures represent mean values.

The experiments are conducted to compare energy efficient resource management algorithm (EERM) with low-energy earliest deadline-first (LEDF) scheduling algorithm proposed by V. Swaminathan and K. Chakrabarty [7] studied scheduling workloads containing periodic tasks in real-time systems. The proposed approach minimizes the total energy consumed by the task set and guarantees that the deadline for every periodic task is met. They present a mixed-integer linear programming model for the NPcomplete scheduling problem. They proposed a low-energy earliest deadline-first (LEDF) scheduling algorithm. The operation of the low-energy earliest deadline first (LEDF) is as follows: LEDF maintains a list of all released tasks, called the ready list. When tasks are released, the task with the nearest deadline is chosen to be executed. A check is performed to see if the task deadline can be met by executing it at the lower voltage (speed). If the deadline can be met, LEDF assigns the lower voltage to the task and the task begins execution. During the task's execution, other tasks may enter the system. These tasks are placed automatically on the ready list. LEDF again selects the task with the nearest deadline to be executed. As long as there are tasks waiting to be executed, LEDF does not keep the processor idle. This process is repeated until all the tasks have been scheduled. In the simulation, we compare EERM with LEDF by varying energy budget and price to study how they affect the performance of two algorithms. To investigate mobile grid settings and proposed energy efficient resource management algorithm, we evaluate them with respect to two criteria: application efficiency and resource efficiency which includes execution success ratio, energy consumption ratio, allocation efficiency and resource utilization. Energy consumption ratio is defined as the percentage of consumed energy among total 


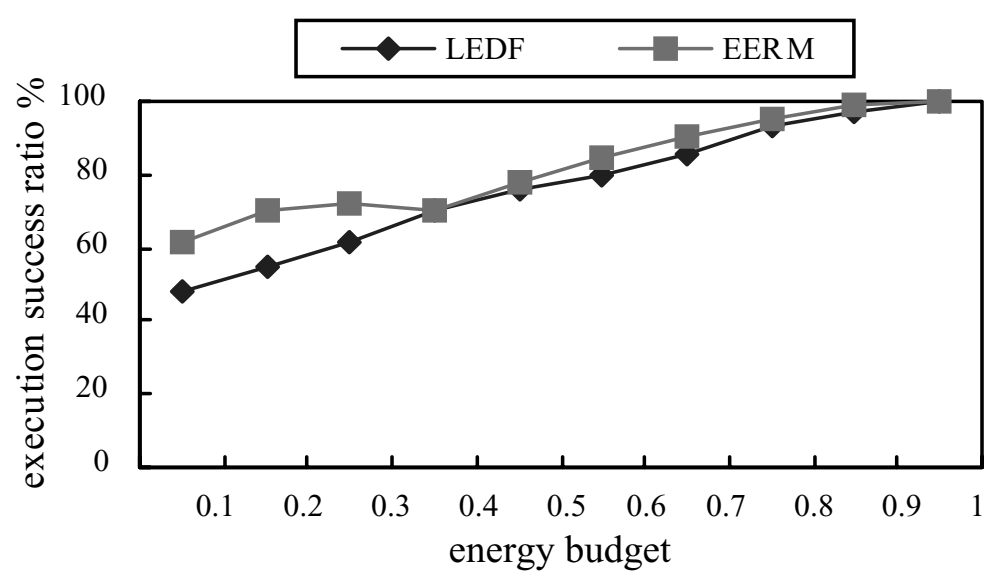

Fig. 1. Execution success ratio under various energy budget.

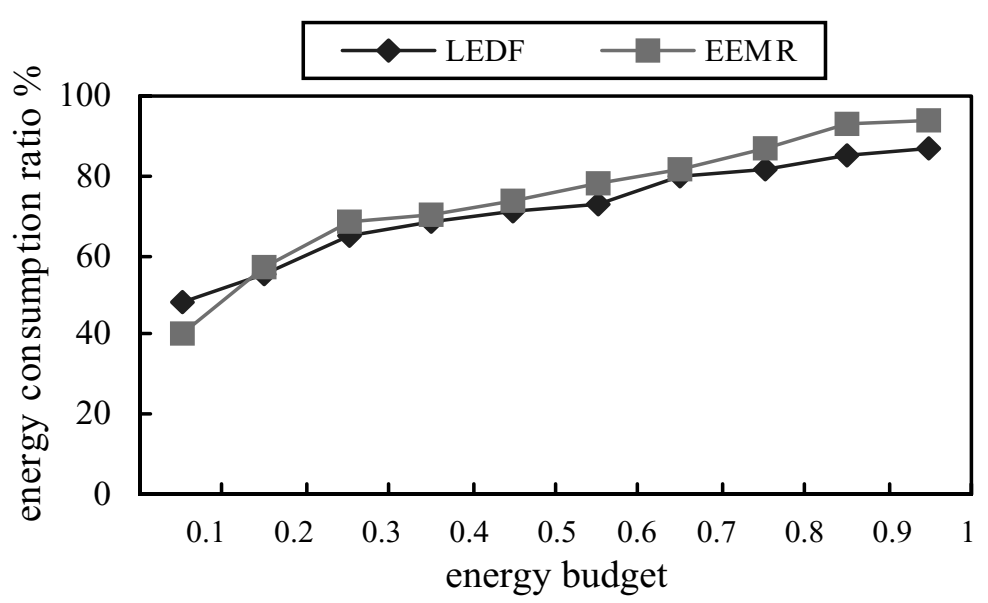

Fig. 2. Energy consumption ratio under various energy budget.

available energy resources. Execution success ratio is the percentage of tasks executed successfully before their deadline. Resource utilization is the ratio of the consumed resources to the total resources available as a percentage, commonly refers to the percent of time a resource is busy. Allocation efficiency is a measure of the efficiency of the allocation process, which is computed using the number of all requests and number of accepted requests.

The effects of energy budget on execution success ratio, energy consumption ratio, allocation efficiency and resource utilization were illustrated in Figs 1-4 respectively. Figure 1 is to show the effect of energy budget on execution success ratio. When increasing energy budget values, the execution success ratio becomes higher. A larger energy budget enables grid user to have enough energy to meet the deadlines and complete the task before its deadline. When energy budget increases $(E=0.8)$, execution success ratio is as much as $30 \%$ more than that with $E=0.3$. Figure 2 shows the energy consumption ratio under different energy budgets. When the energy budget is high, the impact of different energy budget constraint on the energy consumption ratio is obvious; the energy consumption ratio is also high, because grid user tends to choose more energy-consuming resource to complete tasks within deadline. When $E=$ 0.8 , the energy consumption ratio of EERM is $35 \%$ less than $E=0.3$. Under same energy budget $(E=$ 


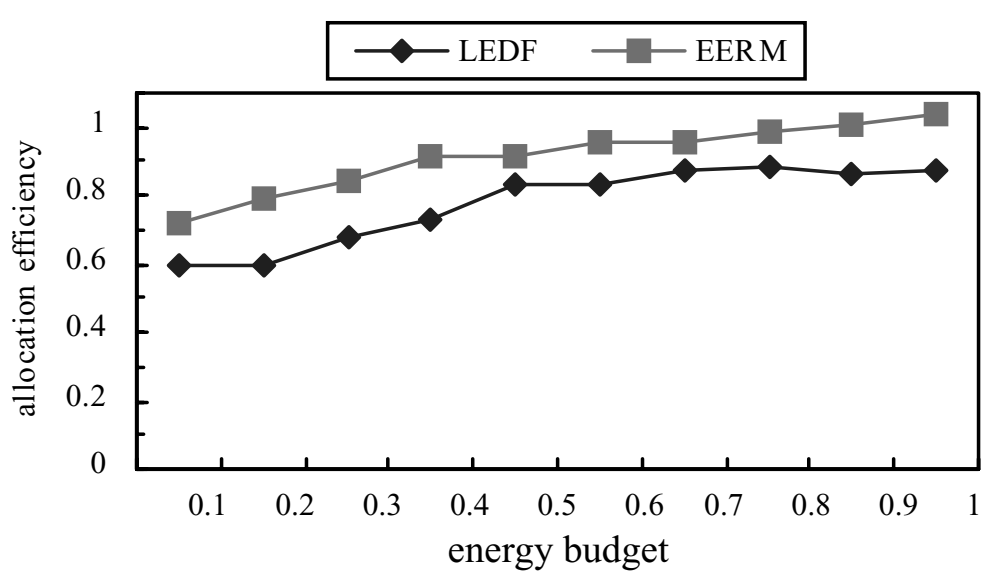

Fig. 3. Allocation efficiency under various energy budget.

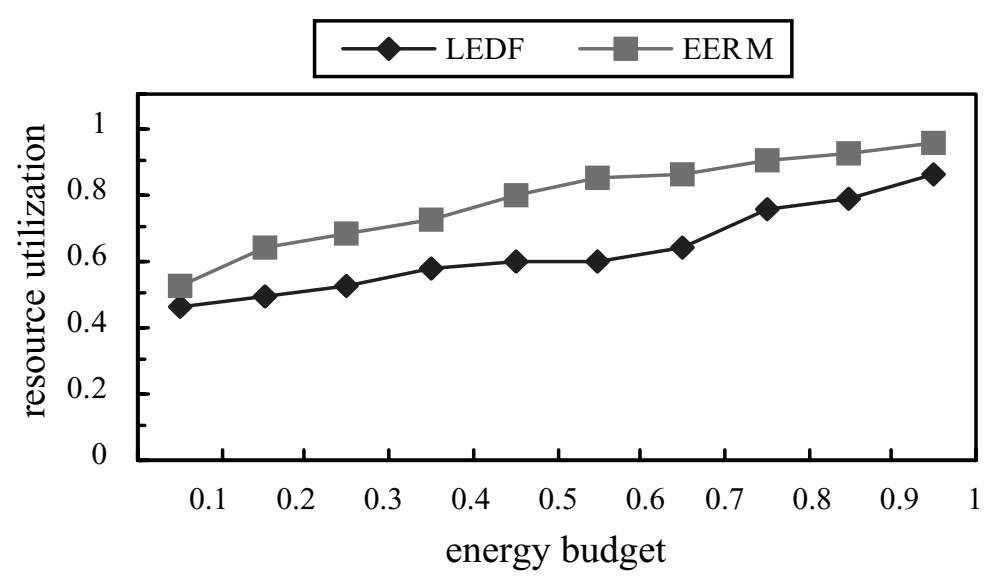

Fig. 4. Resource utilization under various energy budget.

0.3), the energy consumption ratio of LEDF is 6\% less than EERM. Figure 3 shows the improvement of allocation efficiency as energy budget $E$ increases. When the energy budget $E$ is low, the system is very energy-constrained and it is crucial to utilize any excess energy due to achieve the performance objective on time. As energy budget $E$ increases, the system becomes less energy-constrained; more jobs can be executed, the allocation efficiency is increased. When energy budget reaches maximum $(E=100 \%)$, because the system has enough energy to meet all the deadlines and the allocation efficiency reaches its maximum value. Figure 4 shows the resource utilization under different energy budgets. As the energy budget is higher, the resource utilization becomes higher. When $E=0.9$, the resource utilization is as much as $39 \%$ more than utilization by $E=0.2$. Because when the energy budget decreases quickly, the users will be prevented from obtaining energy consuming resources. So, some energy consuming resources will be underutilized.

The impacts of the price on resource utilization, energy consumption ratio, execution success ratio, allocation efficiency were illustrated in Figs 5-8 respectively. The resource price $(p)$ is set from 10 to 500 grid dollars. From the results in Fig. 5, as price is higher, the resource utilization becomes lower. When $p=500$, the resource utilization of EERM is as much as $28 \%$ less than utilization by $p=100$. Because 


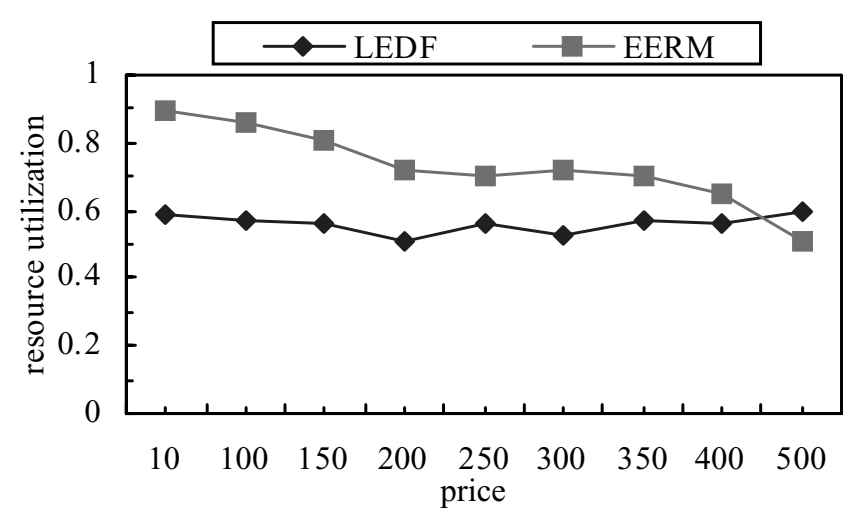

Fig. 5. Resource utilization vs. price.

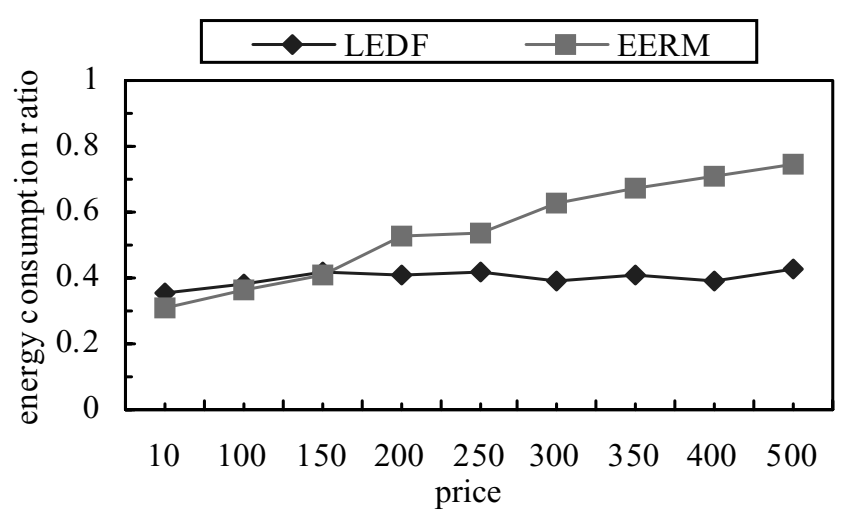

Fig. 6. Energy consumption ratio vs. price.

when the price increases quickly, the users with low expense budget will be prevented from obtaining resources. The smaller is $p$, the lower is the energy consumption ratio as shown in Fig. 6. Because when price becomes high, users will afford more payment to obtain energy-consuming resource, some tasks can't be completed before their deadlines. Price increasing quickly leads to some users with low budget can't be satisfied to fulfill their achievements. When $p=500$, energy consumption ratio of EERM is as much as $34 \%$ more than that by $p=100$. Considering the execution success ratio, the results of Fig. 7 show that when increasing price values, the execution success ratio become lower. Because when price becomes high, grid users will afford more payment to obtain the grid resource, some users with low budget will not complete tasks before their deadlines. When price increases $(p=500)$, execution success ratio of EERM is as much as $39 \%$ less than that with $p=10$. Considering the allocation efficiency, the results of Fig. 8 show that when increasing $p$, the allocation efficiency become lower. Increasing prices of resource provider will prevent users from being admitted by the system, fewer users will exploit the resources. When $p=500$, the allocation efficiency reduce to nearly $42 \%$ compared with $p=10$.

\section{Conclusions}

This paper considers energy efficient resource management in mobile grid. The objective of energy efficient resource management in mobile grid is to maximize the utility of the mobile grid which is 


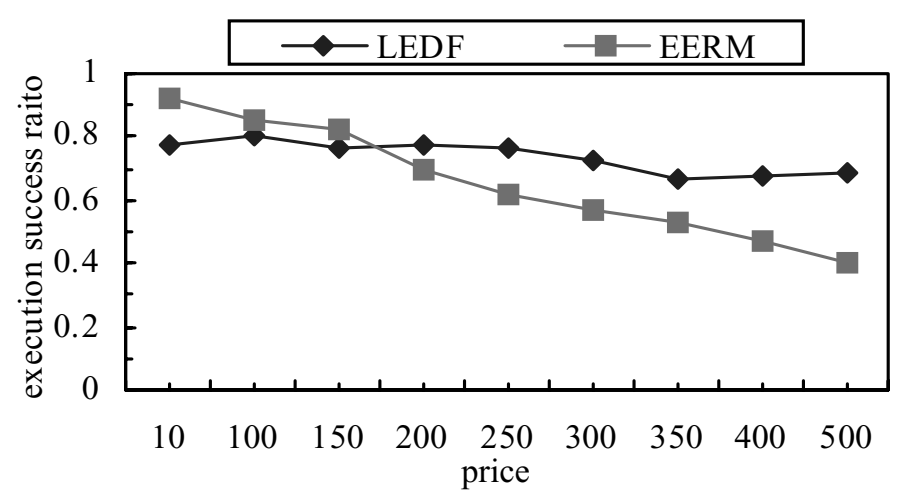

Fig. 7. Execution success ratio vs. price.

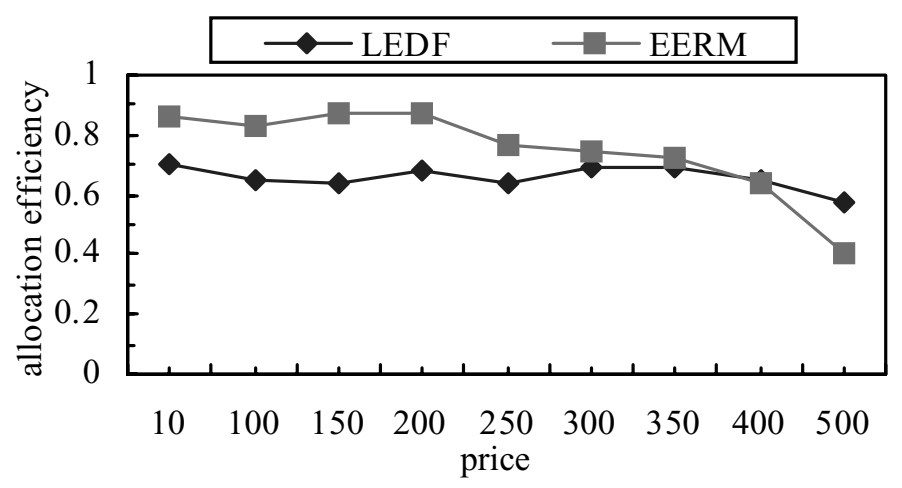

Fig. 8. Allocation efficiency vs. price.

denoted as the sum of grid application utility. The utility function models benefits of application and system. By using nonlinear optimization theory, energy efficient resource management in mobile grid can be formulated as multi objective optimization problem. In order to derive a distributed algorithm to solve global optimization problem in mobile grid, we decompose the problem into the sub problems. The proposed energy efficient resource management algorithm decomposes the optimization problem via iterative method. To test the performance of the proposed algorithm, the simulations are conducted to compare energy efficient resource management algorithm with other energy aware scheduling algorithm.

\section{Acknowledgements}

The work was supported by the National Natural Science Foundation of China (NSF) under grants (No. 60773211, No. 60970064), Program for New Century Excellent Talents in University, China (NCET08-0806), the National Science Foundation of HuBei Province under Grant No. 2008CDB335, the Fundamental Research Funds for the Central Universities (2010-II-003), and Fok Ying-Tong Education Foundation, China (Grant No. 121067).

\section{References}

[1] S.P. Ahuja and J.R. Myers, A Survey on Wireless Grid Computing 37(1) (2006 7), 3-21. 
[2] T. Xie, X. Qin and M. Nijim, Solving Energy-Latency Dilemma: Task Allocation for Parallel Applications in Heterogeneous Embedded Systems, Proceedings of the 2006 International Conference on Parallel Processing (ICPP'06), IEEE Press, 2006.

[3] Y. Huang, S. Mohapatra and N. Venkatasubramanian, An energy-efficient middleware for supporting multimedia services in mobile grid environments, IEEE International Conference on Information Technology, 2005.

[4] Z.L. Zong and X. Qin, Energy-Efficient Scheduling for Parallel Applications Running on Heterogeneous Clusters, International Conference on Parallel Processing (ICPP 2007), IEEE Press, 2007.

[5] T.A. AlEnawy and H. Aydin, Energy-Constrained Scheduling for Weakly-Hard Real-Time Systems, Proceedings of the 26th IEEE International Real-Time Systems Symposium (RTSS'05), IEEE Press, 2005.

[6] K.H. Kim, R. Buyya and J. Kim, Power Aware Scheduling of Bag-of-Tasks Applications with Deadline Constraints on DVS-enabled Clusters, Proceedings of the Seventh IEEE International Symposium on Cluster Computing and the Grid, IEEE Computer Society Washington, DC, USA, 2007, 541-548.

[7] V. Swaminathan and K. Chakrabarty, Real-time task scheduling for energy-aware embedded systems, Journal of the Franklin Institute 338 (September 2001), 729-750.

[8] E. Park and H. Shin, Multimedia Service Composition for Context-Aware Mobile Computing, T.-J. Cham et al., (eds.), MMM 2007, LNCS 4352, Part II, 2007, pp. 115-124.

[9] C. Li and L. Li, Joint QoS Optimization For Layered Computational Grid, Information Sciences, Vol 177/15, pp. 3038-3059, Elsevier, August 2007.

[10] C. Li and L. Li, Agent Framework to Support Computational Grid, Journal of Systems and Software, Elsevier, Vol 70/1-2, pp. 177-187, February, 2004.

[11] C. Li and L. Li, Multi Economic agent interaction for optimizing the aggregate utility of Grid Users In Computational Grid, Applied Intelligence, Springer-Verlag Heidelberg, Vol25/2, pp. 147-158, October, 2006.

[12] C. Li and L. Li, A Distributed Utility-based Two Level Market Solution For Optimal Resource Scheduling In Computational Grid, Parallel Computing, Elsevier, USA, Vol 31/3-4, pp. 332-351, March-April, 2005.

[13] C. Li and L. Li, Utility based QoS Optimisation Strategy For Multi-Criteria Scheduling on the Grid, Journal of Parallel and Distributed Computing, Vol67/2, pp. 142-153, Elsevier, USA, February, 2007.

[14] F. Kelly, A. Maulloo and D. Tan, Rate control for communication networks: shadow prices, proportional fairness and stability, J of Operational Res Soc 49(3) (1998), 237-252.

[15] JAVASIM, http://javasim.ncl.ac.uk.

[16] BRITE, http://www.cs.bu.edu/brite.

[17] N. Chand, R.C. Joshi and M. Misra, Cooperative caching in mobile ad hoc networks based on data utility, Mobile Information Systems 3(1) (2007), 19-37.

[18] M. Aleksy, T. Butter and M. Schader, Architecture for the development of context-sensitive mobile applications, Mobile Information Systems 4(2) (2008), 105-117.

[19] V. Reinhard and J. Tomasik, A centralised control mechanism for network resource allocation in grid applications, International Journal of Web and Grid Services 4(4) (2008), 461-475.

[20] P. Hellinckx, F. Arickx, J. Broeckhove and G. Stuer, The CoBRA grid: a highly configurable lightweight grid, International Journal of Web and Grid Services 3(3) (2007), 267-286.

Li Chunlin received PhD degree in Computer Software and Theory from Huazhong University of Science and Technology in 2003. She now is a professor of Computer Science in Wuhan University of Technology. Her research interests include computational grid, distributed computing and mobile agent. She has published over 20 papers in international journals.

Li Layuan received BE degree in Communication Engineering from Harbin Institute of Military Engineering, China in 1970 and ME degree in Communication and Electrical Systems from Huazhong University of Science and Technology, China in 1982. He academically visited Massachusetts Institute of Technology, USA in 1985 and 1999, respectively. Since 1982, he has been with the Wuhan University of Technology, China, where he is currently a Professor and PhD tutor of Computer Science, and Editor in Chief of the Journal of WUT. He is Director of International Society of High-Technol. and Paper Reviewer of IEEE INFOCOM, ICCC and ISRSDC. He was the head of the Technical Group of Shaanxi Lonan P.O. Box 72, Ministry of Electrical Industry, China from 1970 to 1978. His research interests include high speed computer networks, protocol engineering and image processing. Professor Li has published over 150 technical papers and is the author of six books. He also was awarded the National Special Prize by the Chinese Government in 1993. 

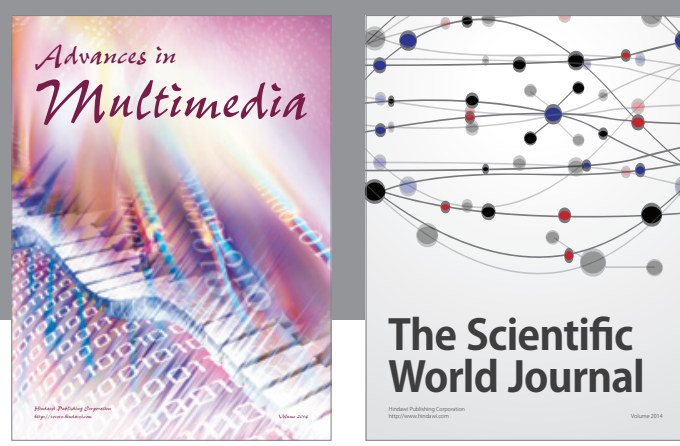

The Scientific World Journal
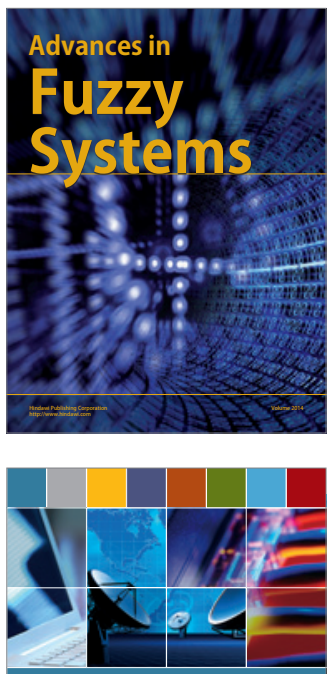

Computer Networks and Communications
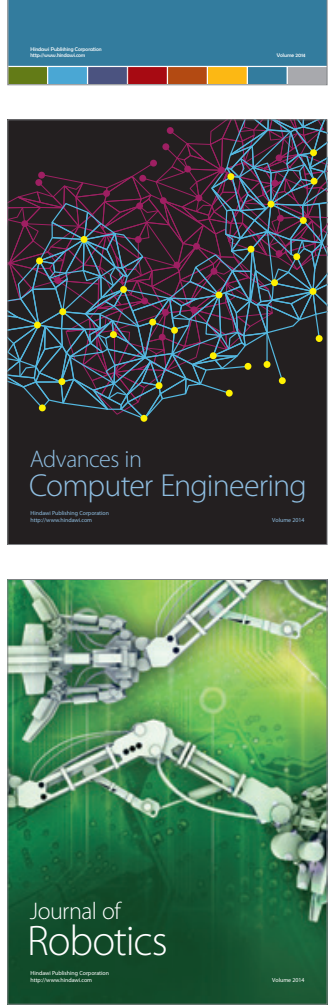
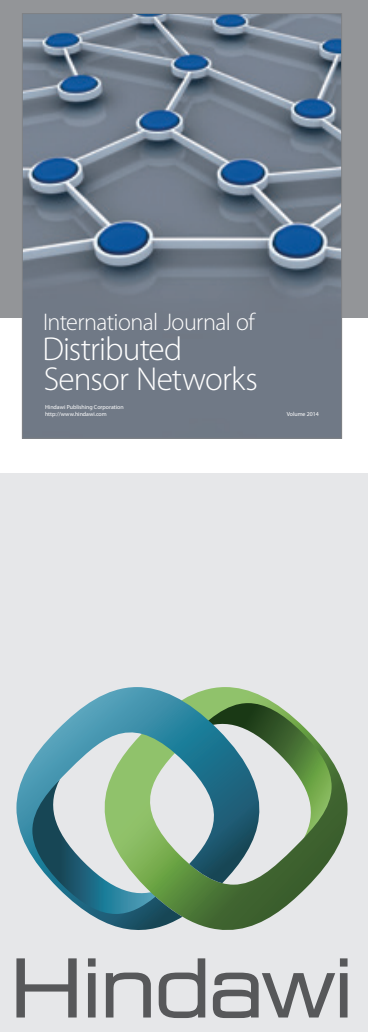

Submit your manuscripts at

http://www.hindawi.com
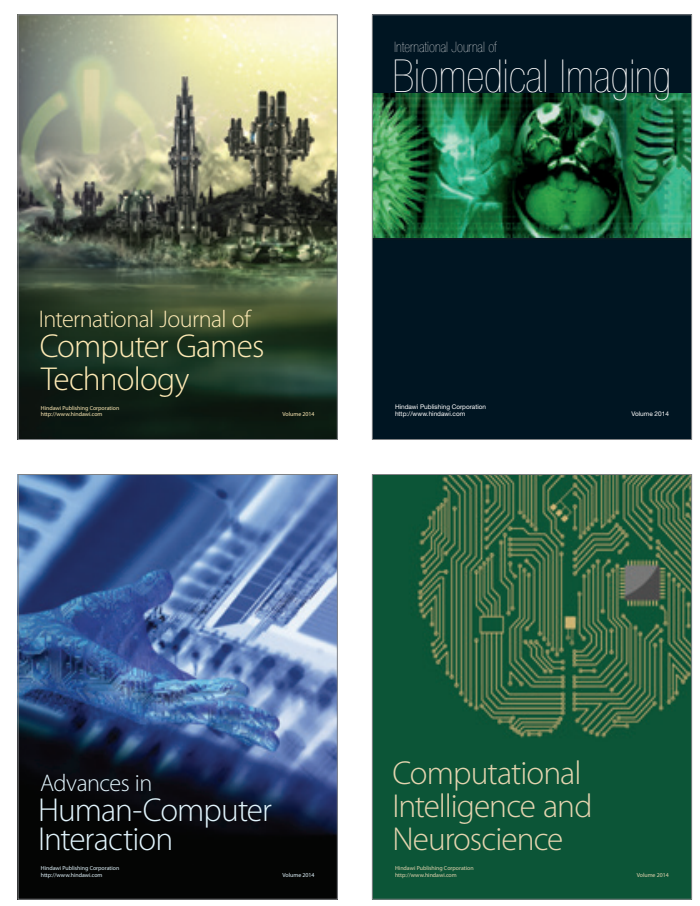
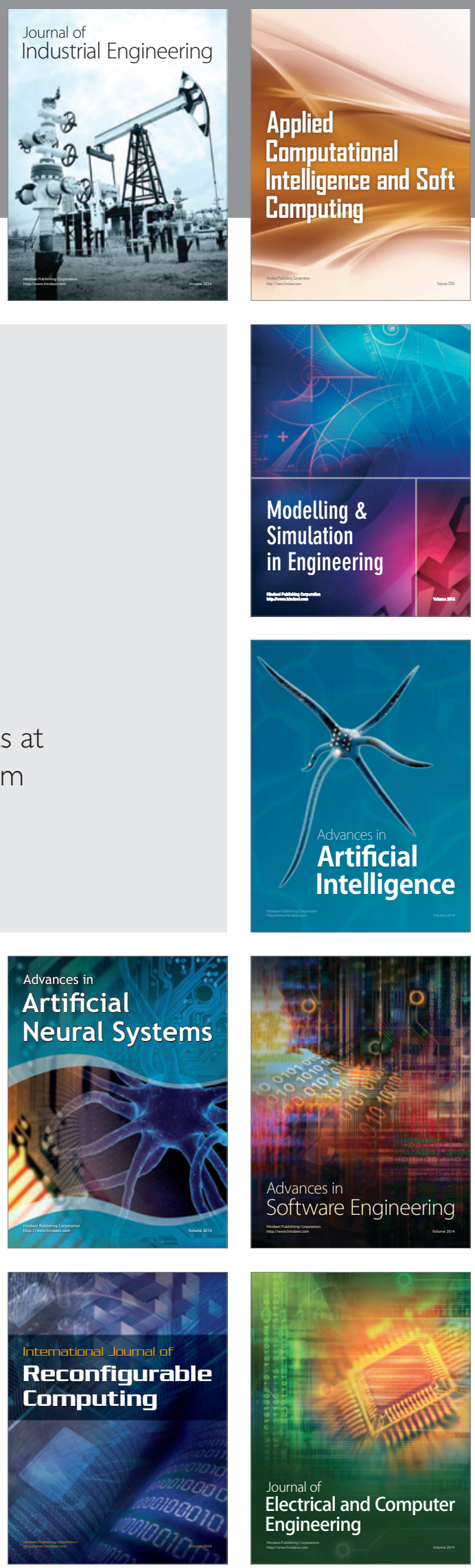